\title{
An Alternative Cosmology to the Big Bang-Dispersive Extinction Theory of Red Shift
}

\author{
Ling Jun Wang ${ }^{1}$ \\ ${ }^{1}$ Department of Physics, Geology and Astronomy, University of Tennessee at Chattanooga, Chattanooga, USA \\ Correspondence: Ling Jun Wang, Department of Physics, Geology and Astronomy, University of Tennessee at \\ Chattanooga, Chattanooga, TN 37403, USA. Tel: 1-423-425-5248. E-mail: lingjun-wang@utc.edu
}

Received: January 14, 2013 Accepted: February 21, 2013 Online Published: March 21, 2013

doi:10.5539/apr.v5n2p47

URL: http://dx.doi.org/10.5539/apr.v5n2p47

\begin{abstract}
A brief review is given to the fundamental difficulties facing the Big Bang Theory, with the emphases on the geocentric nature, the extreme violation of the conservation laws of mass and energy, the violation of Maxwell's velocity distribution, the horizon problem, the extreme instability and the dark matter problem. The historical alternatives and their problems are reviewed. The DET is then presented as a new alternative interpretation of the cosmic redshift, and therefore, as a new alternative to the Big Bang cosmology. The existing experimental evidences to vindicate or falsity DET versus Big Bang theory are discussed. Experiments for detailed tests of the DET-the redshift is proportional to the square of the linewidth and inversely proportional to the cube of the wavelength-are also proposed.
\end{abstract}

Keywords: DET, Big Bang, redshift, cosmology, alternative cosmology, infinite universe

\section{Introduction}

The Big Bang theory has dominated cosmology for decades. Many of its extraordinary concepts have permeated into the blood vessels of young children through fascinating si-fi movies interweaved with oriental martial arts and ex-terrestrial romance. To many scientists the theory of Big Bang is the orthodox physical science. The Big Bang theory tells an incredible story of creation of the universe ex nihilo. However, the Big Bang theory has some fundamental difficulties, the most important being the extreme violation of the conservation laws of mass and energy, the extreme violation of the principle of relativity, the dramatic violation of Maxwell's speed distribution, the geocentric nature and the extreme instability of the model.

The multitude of fundamental inconsistencies of the Big Bang theory motivated physicists to propose alternative theories, the most noteworthy being the tired-light cosmology (Zwicky, 1929), the steady-state cosmology (Bondi, 1948, 1960; Hoyle, 1948), the plasma universe (Alfven, 1966; Klein, 1971; Lerner, 1991), and the theory of discordant redshift association (Field et al., 1973; Arp, 1987, 1990, 2003). These alternatives did not gain much momentum, partially due to the fact that these alternatives were also based on some sort of ad hoc hypotheses.

Recently, a Dispersive Extinction Theory (DET) of the cosmic red shift was proposed by this author in a number of publications (Wang, 2005, 2007, 2008, 2011). DET interprets the cosmic redshift as the result of dispersive extinction (absorption and scattering) of the star light by the intergalactic space medium during its propagation towards the earth. The theory is based on the same phenomenon that is responsible for the star-reddening and for the sky to be blue. Since the space medium absorbs and scatters the blue light more than it does the red component, the Gaussian peak of a spectral line would shift towards the red side, resulting in a spectral redshift. No global galactic movement is needed in this theory to explain the cosmic redshift. As a result, DET allows a stable non expanding universe, infinite in space and time. The new theory is free of all the problems intrinsic to the Big Bang Theory.

Not only DET offers an alternative cosmology, it also contains rich physics never known to astrophysics community before: DET predicts that the cosmic redshift is proportional to the square of the linewidth and inversely proportional to the cube of the wavelength. The dependence on linewidth and wavelength may be used to vindicate or falsify DET. Besides, DET also offers an interpretation of the abnormal redshifts of the quasars: The incredibly high redshifts of the quasars may well be due to the large line widths typical of the quasars. The 
linewidth and wavelength dependence also indicate possible erroneous identification of the redshifts in the redshift measurements.

\section{Fundamental Inconsistencies of the Big Bang Theory}

\subsection{The Geocentric Nature of Hubble Law}

In 1920s, Hubble discovered that the spectroscopic red shift of a star or galaxy is by and large linearly proportional to its distance from the earth (Hubble, 1926, 1929, 1936). Hubble proposed that the red shift was caused by Doppler Effect due to the receding movement of the stars and galaxies. Such interpretation logically led to an ever expanding universe. Tracing back this expansion into the remote past, it leads to a creation of the universe from nowhere out of nothing at certain time origin. The event of creation is called the Big Bang.

An expanding universe with all heavenly bodies moving isotropically away from the earth suggests a geocentric theory which is patently false. To defend the Big Bang theory from such critics, it was argued that if the universe is expanding linearly from the singularity, the heavenly bodies would appear to be leaving away from each other with isotropic velocity distribution with respect to any observer, including the observer on earth. Such argument is known as the raisin-pudding model or balloon-model: If the pudding is uniformly and linearly expanding, with respect to any individual raisin, which plays the role of a galaxy, all other raisins are leaving isotropically from it.

But there are flaws in such argument. In order for the raisin-pudding model to work, three necessary conditions must be satisfied: 1) Hubble law must be strictly linear; 2) Classical Galileo velocity transformation must apply; 3) The positions and the velocities of the galaxies in the universe must be measured simultaneously. None of these three conditions is met. The raisin-pudding model is nothing more than a gimmick.

First, the linearity of Hubble law is by no means a fact beyond any doubt. As a matter of fact, the plot of redshift-magnitude almost fills up the entire first quadrant. A brute-force fitting of such data into a linear function yields too big uncertainty which suggests that such linear fitting does not make much sense. Up to now, we do not know the Hubble constant within a factor of two. This constant is believed to be anywhere from 35 to $100 \mathrm{~km} \mathrm{~s}^{-1} \mathrm{Mpc}^{-1}$ (Tammann \& Sandage, 1985; Sandage \& Tammann, 1990; de Vaucouleurs, 1993). It is well known that for large values of the red shift the connection between the redshift and the velocity of the galaxy is no longer linear. The non linearity is attributed to a number of possibilities, such as the difficulties in accurate determination of stellar distances, the modification of the inverse-square law relating brightness to distance in a curved space time, the decrease of the energy of the light brought about by the reduction of the frequency of the light wave, the evolution in the luminosity of galaxies with time, etc. (Ohanian, 1976). There is no general agreement about the corrections needed to be made for the non linearity of Hubble law.

In 1992, Segal and Nicoll provided a direct challenge to the linear Hubble law with statistical analysis of data of more than 2000 galaxies (Segal \& Nicoll, 1992). The statistical analysis has convincingly proven that the cosmic red shift is proportional to the square of the distance. Textbook presentations of Hubble law typically report measurements on bright cluster galaxies only. The samples are often subjectively selected from the catalog of Abell (1958) which explicitly assumes Hubble law in its selection criterion. The sample of Hoessel et al., which provides one of the major supports for Hubble's law, consists of 116 galaxies drawn entirely from the Abell catalog (Hoessel et al., 1980).

Second, the classical Galilean velocity transformation does not apply to the scenario of Big Bang since the universe is supposedly expanding with relativistic velocity or faster than the speed of light (see next section). Wang has shown rigorously that if relativistic velocity transformation is applied, the isotropy of Hubble law leads inevitably to the conclusion that the earth is at the center of the Big Bang expansion (Wang, 2007).

Finally, the positions and the velocities of the different galaxies could in no way be measured simultaneously by an observer on the earth due to the limit of speed of light. The information of the position and velocity of a galaxy 10 billion light years from us ware sent to us 10 billion years ago. Likewise, the information of the position and velocity of a galaxy 5 billion light years from us ware sent to us 5 billion years ago. The difference in the time of propagation can differ by billions of years! It is simply impossible to make the measurements simultaneous. Wang has shown rigorously that, even we allow the linear Galilean velocity transformation and accept the linearity of Hubble law, the impossibility of simultaneous measurement of positions and velocities of galaxies inevitably leads to a geocentric universe if the Big Bang interpretation of Hubble law is adopted (Wang, 2007).

The geocentric nature of the Big Bang theory is also evidenced by the abnormal redshifts of the quasars. The great redshifts would place all quasars at the edge of the Big Bang universe. Placing these objects at the edge of 
the universe would mean that the absolute brightness of quasars is unbelievably high, about a billion times as bright as our Milky Way. But the period of the brightness fluctuation of the quasars can be as short as a year, which means that the diameters of the quasars are in the order of light year based on causality consideration. This is about $10^{-5}$ of the diameter of the Milky Way. The unbelievably high brightness and small size give an estimated mass density of the quasars to be 14 orders of magnitude greater than that of the Milky Way. Namely, the quasars belong to a distinctively different type of objects from the regular galaxies. It means that the universe is denser at the edge populated by quasars, and we are equally distant from this layer of skin of the universe. It would mean that we are at the center of the universe. The situation is similar to a bug in a spherical melon who finds itself equally distant from the skin of the melon (See Figure 1). Such geocentric picture cannot be easily brushed away with arguments like the raisin-pudding model.

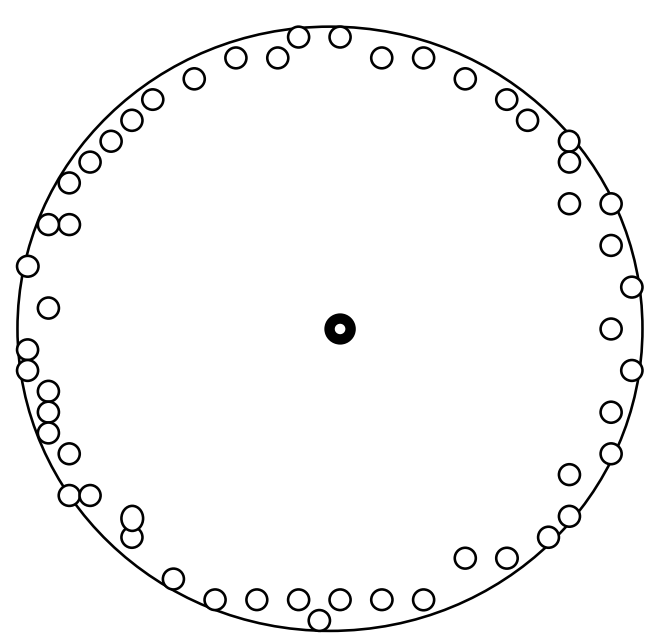

Figure 1. The quasar distribution

According to the Big Bang theory, all quasars, represented by the small circles near the edge, will be near the edge of the universe. Such interpretation would imply that the quasars are extraordinarily massive with brightness of about one billion times that of the Milky Way, forming a "hard crust" of the universe. It destroys one of the most fundamental hypotheses of the Big Bang theory-the homogeneity hypothesis. More seriously, it leads to a geocentric theory since the quasars are observed to be isotropically distributed about the earth (the dark circle at the center of the universe).

\subsection{The Extreme Violation of the Conservation Laws of Mass and Energy}

The notion of having the enormous mass and energy of the whole universe created ex nihilo is an extreme violation of the conservation laws of mass and energy. One may not necessarily take the creation stories of "ultimate free lunch", "energy financing" or "quantum foam theory" at their face value, but the scientific community is lead to generally believe that the fluctuation of vacuum would create energy, based on Heisenberg's uncertainty principle, which would in turn be converted to mass, based on Einstein's mass-energy relationship. Therefore, the conservation laws of mass and energy are trumped by the principle of uncertainty. The question is: which is more fundamental? The conservation laws of mass and energy, or the uncertainty principle?

We all know that the conservation laws of mass and energy are the most fundamental laws of science that was obtained though numerous repeated macroscopic experiments and engineering practices. If this law is upset, not only physics but the entire scientific edifice will collapse. Little needs to add to the importance and fundamentality of the conservation laws of mass and energy.

As to the Heisenberg's uncertainty principle, it is merely an algebraic result from the non relativistic quantum mechanics, which says that the product of the uncertainties in position and momentum measurements is greater than the Plank constant, so is the product of the uncertainties in time and energy measurements. Apparently, the Uncertainty principle is only a relationship about the accuracy of measurements. It does not say anything about 
creation at all. If error in measurement means creation, we would then have a solution to the energy crises: One needs only to measure a tank of gasoline with a lousy measuring device to create gasoline. Likewise, one can measure gold bullions to create gold and overcome the economic crisis.

The creation of mass from vacuum was never truly observed experimentally. No "pair production" of electron and positron would takes place without presence of a target, or the emulsion detector, or moisture molecules in a cloud chamber and the pre-existence of enough photon energy. No pair production has ever been observed in vacuum, no matter how much energy the photons might have. (The minimum is about $1 \mathrm{MeV}$.) The electron-positron pair is produced from a photon-target interaction (with presence of both matter and energy) rather than created ex nihilo. The preexistence of energy for pair-production is a common knowledge, but the preexistence of matter (target, emulsion or moisture molecules) is not well recognized by the general community.

As to the creation of energy ex nihilo, it has been proven impossible by numerous failures of inventing the perpetual machine, and by the daily routines of science and engineering practices. A theory based on creation of the enormous mass and energy of the entire universe is a fundamental violation of the physical laws and a serious contradiction against reality.

\subsection{Violation of the Principle of Relativity-the Horizon Problem}

The geocentric nature of the Big Bang theory discussed above is in essence a fundamental violation of the principle of classical relativity which denies the earth as the center of the universe. Big Bang theory also has a well-known horizon problem that violates Einstein's principle of relativity-Nothing could move faster than photon. If the speed of an object is equal to the speed of light, the Lorentz transformation matrix would become infinity, so would the metric tensor elements. If the speed is greater than the speed of light, the space and time would be reversed, i.e., the time would become 3-dimensional and the space would become 1-dimensional. However, the speed of the Big Bang during the decoupling period is hundreds times greater than the speed of light. The decoupling epoch was at about $3 \times 10^{5}$ years after the Big Bang, but the radius of the universe at this time was about $7 \times 10^{7}$ light years according to the Big Bang Theory. The velocity of expansion in this period is then

$$
v=\left(7 \times 10^{7} \mathrm{LY}\right) /\left(3 \times 10^{5} \mathrm{Y}\right)=233 \mathrm{c} !
$$

This problem is known as the "horizon problem". To solve the horizon problem, Allen Guth proposed in 1980s an Inflation Theory, which says that the universe went through a inflation in the time period from $10^{-36} \mathrm{~s}$ to $10^{-33}$ s. During this short time period the size of the universe was inflated from $10^{-65} \mathrm{LY}$ to $10^{-15} \mathrm{LY}(30 \mathrm{~m})$. The velocity of "Inflation":

$$
v_{i}=\left(10^{-15} \mathrm{LY}\right) / 10^{-33} \mathrm{~s}=10^{26} \mathrm{c} \text { ! }
$$

The speed of inflation was 26 orders of magnitude greater than the speed of light! It is an extreme violation of the theory of relativity. Violation of relativity is a logical paradox of the Big Bang theory since it is built upon relativity.

\subsection{The Violation of Maxwell Velocity Distribution}

The Big Bang theory employs much of thermodynamics to construct a picture of universe as an expanding fireball. One expects the fireball of Big Bang to be a thermodynamic ensemble that requires all the particles in the system to collide numerous times to reach thermodynamic equilibrium. However, the Hubble law says that the particles at larger distance from the singularity move faster than the ones at shorter distances. The ones falling behind could never catch up with the ones in front of them, and no collision is possible. The radial expansion does not allow angular collision either. The fireball of the Big Bang does not qualify for a thermodynamic ensemble, period. Many thermodynamic concepts, such as temperature, which is the average (as a result of collision) kinetic energy of molecules, and the pressure, which requires collisions to transfer momentum, simply have no mining for a collection of particles without collision. As a result, the laws of thermodynamics do not apply in Big Bang theory. The temperature of a Big Bang fireball simply does not make any sense.

A way of looking at the violation of thermodynamics by the Big Bang theory is to compare the speed distribution of the particles in the system. If the system is a thermodynamic ensemble, it should obey the energy partition principle and Maxwell's velocity distribution: 


$$
F(v)=4 \pi n\left(\frac{m}{2 \pi k T}\right)^{\frac{3}{2}} v^{2} \exp \left[-\frac{m v^{2}}{2 k T}\right]
$$

where $F(v)$ is the mean number of molecules with speed $v$ per unit volume per unit speed interval, $m, k, T$ are, respectively, the mass of the molecule, the Boltzmann constant and the temperature. $n$ is the number density of molecules. The distribution Equation (1) is plotted in Figure 2.

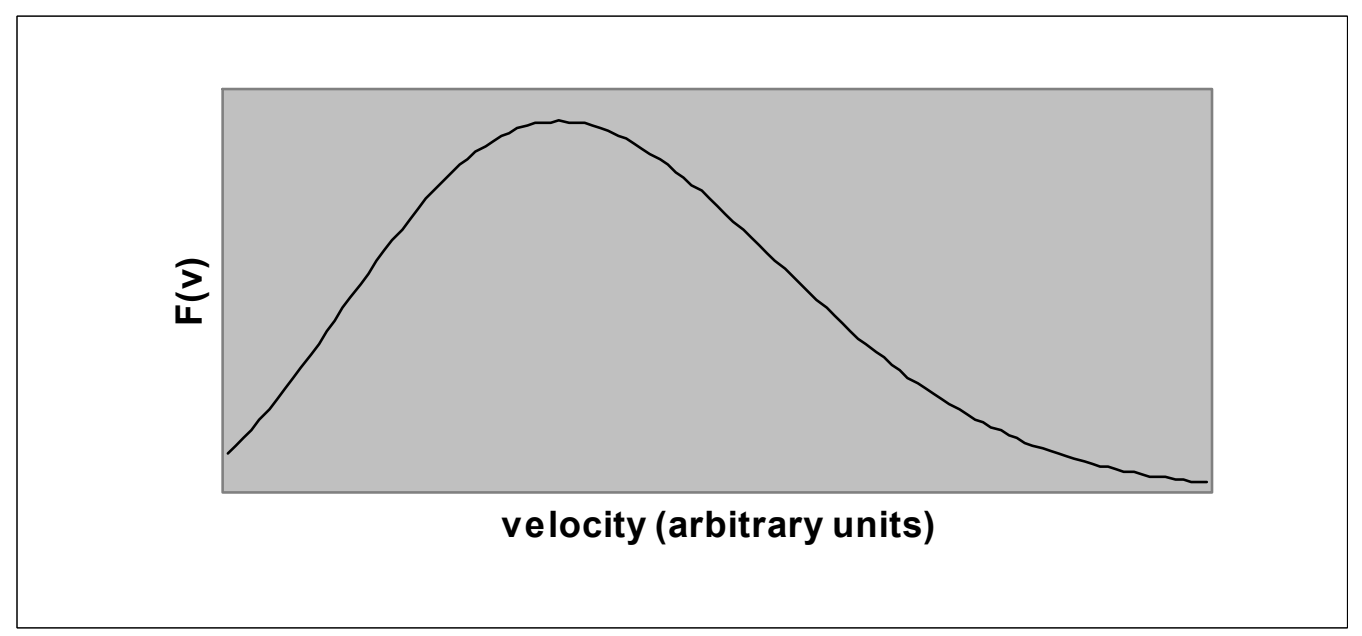

Figure 2. Maxwell velocity distribution of thermodynamic ensemble

For systems not at equilibrium, (for example, a nuclear explosion), the velocity distribution may deviate from Maxwell velocity distribution function to some extent, but qualitatively the velocity distribution of any natural system should show two important characteristics similar to the Maxwell distribution: a) Most of the mass should be lumped around certain finite average velocity; b) The distribution function approaches zero when the velocity of particles approaches either zero or infinity. Namely, the distribution function should show a shape of asymmetric bell. But the Big Bang theory presents a dramatically different velocity distribution of the universe. This dramatic distribution can be readily derived from the fundamental postulations of the Big Bang theory. Thus, the total mass of the universe is equal to the integration of mass density over the volume:

$$
M=\iiint \rho d V=\iiint \rho r^{2} d r d \Omega
$$

where $\mathrm{m}, \rho, V, r$ and $\Omega$ are, respectively, the total mass, the mass density, the volume, the radius and the solid angle. According to the Big Bang theory, the universe is homogeneous and isotropic, the mass density is a constant. The integration over the solid angle is $4 \pi$. We then have

$$
M=4 \pi \rho \int r^{2} d r
$$

According to the Hubble law, the distance is proportional to the velocity:

$$
v=c H r
$$

Substituting Equation (4) into Equation (3) yields

$$
M=\int f(v) d v=\int \frac{4 \pi \rho}{c^{3} H^{3}} v^{2} d v
$$


The integrand gives the velocity distribution of mass of the Big Bang universe:

$$
f(v)=\frac{4 \pi \rho}{c^{3} H^{3}} v^{2}
$$

This quadratic function is shown in Figure 3, which is dramatically different from Maxwell's velocity distribution shown in Figure 2. The violation of Maxwell's velocity distribution demonstrates vividly that the Big Bang universe is not a thermodynamic system, and makes the applicability of thermodynamics to the Big Bang theory highly dubious.

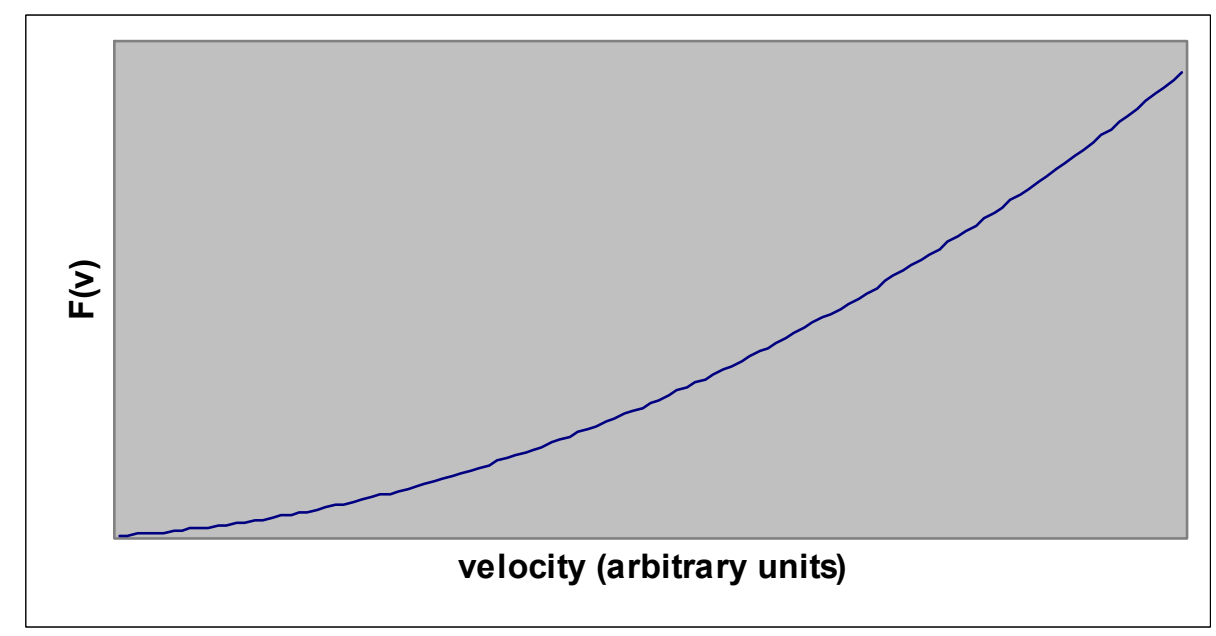

Figure 3. The velocity distribution of Big Bang which is dramatically different from the velocity distribution of thermodynamic ensemble (Figure 2)

\subsection{Extreme Instability of the Big Bang Model}

If a mathematical model is sensitive to certain fitting parameter, it is an indication that the model is not stable, and therefore, not a good one. The more sensitive it is, the less credible is the model.

During decades long history of Big Bang cosmology, about half dozen different models of Big Bang theory phased in and out, all depending on a crucial parameter-the density parameter $\Omega$, which is the ratio of the mass density of a model to what is called the "critical mass density", at which the space-time is flat. Namely, the space-time is flat when $\Omega=1$. Any infinitesimal deviation of $\Omega$ from unity would result in a curved space-time. If $\Omega>1$ in a model, the universe is "closed" and would eventually die in a "Big Crunch". If $\Omega<1$, however, the universe is "open" and would be diluted into nothing in the remote future. Calculations show that the mass density parameter $\Omega$ cannot deviate from unity by more than $10^{-58}$ in order for the universe to survive 15 billion years since its creation predicted by the Big Bang theory. If the mass density deviates from unity by $10^{-40}$, the universe would die in less than a second. Such unbelievable fine tuning of a fitting parameter manifests extreme instability of the theory.

\subsection{The Mystery of Dark Mater}

Since the Big Bang theory needs the mass density to be almost exactly unity, the mass of the universe must be equal to the critical mass of $10^{-26} \mathrm{~g} / \mathrm{c} . c .$. Unfortunately (or fortunately), the observed average mass density of the universe is merely a humble $3 \%$ of that predicted, or needed, by the Big Bang theory. It is a significant and fundamental failure of the theory that overshadows all other claimed successes.

The embarrassment was dismissed politically: The Big Bang cosmologists simply declare that $97 \%$ of the predicted mass is the "dark matter", which cannot be observed because it is "dark". A theoretical failure is thus politically transformed into a great discovery-the dark matter-and a reason for further funding.

There is a general misunderstanding of the term "dark matter" among the public. To a layman it seems quite understandable that if something is dark and invisible, then it is unobservable. But the matter of fact is that all dark objects in the space can be, and have been, observed by astrophysicists. The method is quite simple and straightforward. One needs only to measure the distance from a star at the edge of a galaxy and its orbiting 
velocity about the center of the galaxy. The total mass of the galaxy, including both bright objects and dark objects, can be easily calculated according to Newton's law of gravitation:

$$
M=\frac{v^{2} r}{G}
$$

where $M, v, r$ and $G$ are, respectively, the total mass inside the orbit of the star been measured, the velocity and the distance of it from the galactic center, and the gravitational constant. The experimentally observed average mass density of the universe actually includes the dark matter such as the cold meteorites, dusts and gases. The real dark matter is observable, and it is already observed by astrophysicists.

The dark matter has been observed since 1930s. In 1933, Zwicky proposed such dark matter to account for the "missing mass" to explain the orbital velocities of galaxies in clusters (Zwicky, 1933) according to Equation (7). In the late 1960s and early 1970s, Vera Rubin worked with a new sensitive spectrograph that could measure the velocity curve of edge-on spiral galaxies to a greater degree of accuracy (Rubin, 1970) and announced in the 1975 meeting of the American Astronomical Society that most stars in spiral galaxies orbit at roughly the same speed, which implied that the mass densities of the galaxies were uniform well beyond the regions containing most of the stars (the galactic bulge). Rubin's observations and calculations showed that most galaxies must contain about six times as much "dark" mass as can be accounted for by the visible stars.

But these observed dark matters are called the "baryonic dark matters" which are actually included in the current value of the observed average mass density of the universe. The so-called "dark matter" of the Big Bang theory must be something that cannot be detected by Equation (7). Namely, there "dark matters" of the Big Bang must be "dark" to the gravitational field, i.e., it does not obey the law of universal gravitation. It means "gravitationally dark", not "optically dark". But then the problem comes. If the "dark matter" is gravitationally dark, it will not be able to keep the space-time asymptotically flat, which defeats the very purpose of postulating a mass density parameter of unity. The hypothesis of a gravitationally dark "dark matter" is a logical paradox.

The great discrepancy between the observed mass density and the one predicted (or needed) by the Big Bang theory has to be accounted for by the so-called "non-baryonic dark matter" such as the WIMPS (Weakly Interacting Massive Particles), neutralinos, axions, sterile neutrinos, Gravitinos, photinos and may be other supersymmetric particles (Davis et al., 1985).

There are active efforts to detect the so-called "cold dark matters" (CDM). Direct detection experiments are usually set up in deep underground laboratories to reduce the background from cosmic rays. The well known facilities include: the Soudan mine; the SNOLAB underground laboratory at Sudbury, Ontario (Canada); the Gran Sasso National Laboratory (Italy); the Canfranc Underground Laboratory (Spain); the Boulby Underground Laboratory (UK); and the Deep Underground Science and Engineering Laboratory, South Dakota (US). In 2009, the CDMS Collaboration reported two possible WIMP events. They admitted that "this analysis cannot be interpreted as significant evidence for WIMP interactions, but we cannot reject either event as signal." (Ahmed et al., 2009).

Indirect detection experiments are designed to search for the products of WIMP annihilation or decay (Bertone \& Merritt, 2005). If WIMPs are their own antiparticles, then two WIMPs could annihilate to produce gamma rays or particle-antiparticle pairs. Additionally, if the WIMPs are not stable, they could decay into other particles. These processes could be detected indirectly through an excess of gamma rays, antiprotons or positrons emanating from the dark matters. The detection of such a signal is not conclusive evidence for dark matter, because it is buried in gamma rays from other possible sources. (Bertone et al., 2005; Bertone \& Merritt, 2005).

It is also hoped that WIMPs could be produced by man in the large facilities such as the Large Hadron Collider (LHC). By definition, the WIMP has negligible interactions with matter, it may be detected only indirectly if and only if the background collision products are detected and subtracted (Kane \& Watson, 2008). These experiments could show that WIMPs can be created, but it would still require a direct detection experiment to show that they exist in sufficient numbers in the galaxy to account for the dark matter needed by the Big Bang theory.

\subsection{Quantum Gravity}

As creationism is very offensive to the physics community, the Big Bang cosmology is always obliged to answer the inconvenient questions like "where did the enormous mass and energy come from?" and "what was it like before the Big Bang?" A new theoretical trend-quantum gravity-seems to be trying to answer those questions.

The idea of combining the principles of supersymmetry and general relativity, called "supergravity, came into being in the 1970s, but the calculations were too complicated that it may never be provable. In 1980s, Green and 
Schwarz proposed a revolutionary "superstring theory" which attracted much attention of physics community (Green \& Schwarz, 1984). "Superstring theory" needs ten dimensions to work, with 6 "extra dimensions" "curled up" into strings wrapped up on the scale of the Planck length. Such theory is also known as "theory of everything" because it might be a candidate to unify all the fundamental forces. The superstring theory is frustratingly abstract which is hard to be related to physical reality. No one could even explain the physical meaning of the extra dimensions. Moreover, at least five different superstring theories have been developed to compete each others.

The embarrassment seemed to be cleared in 1995 when Whitten (1995) conjectured that five different versions of string theory might be the same theory. If the eleventh dimension is introduced, the different superstring theories might be the different ways of looking at the same thing. With the eleventh dimension added, the "string" becomes "membrane" or "brane"; the "superstring theory" evolves into a "membrane theory", or "M-theory", the complete structures of which had not yet been discovered. The M-theory is compatible with the concept of multiverse. In such a theory, a Big Bang is caused by chance collisions between rippling membranes, and our universe came from a Big Bang which was just one of the many collisions between membranes. The "chance collisions", or parallel universes, might be infinite in number. Such a theory of "creation by chance collision between infinitely many membranes" seems to conjure away the inconvenient and intractable questions of "how was our universe created" and "what was it like before the creation". The constant creation of universes by chance collision between infinitely many membranes simply reduces the status of "universe" into something like "super galaxy". The M-Theory is not the only proposal for a "theory of everything". A "loop quantum gravity" (LQG) was developed by Ashtekar (1986, 1987, 1998, 2004), Ling (Ling \& Smolin, 1999), Smolin (2006, 2010), Rovelli $(1988,1990,1995,1996)$, Thiemann $(2003,2006)$ and others. In LQG the space-time is fundamentally discrete and quantized. Space can be viewed as a network of finite loops called "spin networks", having the size of Planck length (about $10^{-35} \mathrm{~m}$ ). The Loop quantum cosmology (LQC) studies the early universe and the physics of the Big Bang. According to such study, the Big Bang was the cosequence of the last Big Crunch. The terminal Big Bang is replaced by a picture of Big Bounce similar to the picture of oscillating universe.

Both the M-theory and Loop quantum gravity have their fumdamental issues to solve before being accepted into the main stream standard model. For M-theory, the extra dimensions have no empirical support and physical meaning. No physical quantity that is experimentally verifyible has been predicted by the theory. As to the Loop quantum gravity, the proposition of discrete space-time is quite radical.

Before the theorists settle their fundamental issues, we can meke a general comment on their impact on the Big Bang cosmology. Even if one of the theories of quantum gravity has solved its fundamental issues, it could at most help the Big Bang cosmology by offering a plausible answer to the question of creation, it will not be able to resolve other fundamental issues of the Big Bang theory such as the geocentric nature, the horizon problem, the violation of Maxwell velocity distribution, and the stability problem.

\section{Alternative Theories of Cosmology}

The multitude of fundamental difficulties facing the Big Bang theory motivated physicists to propose alternative theories since the very early days of the Big Bang cosmologyy, the most noteworthy being the tired-light cosmology (Zwicky, 1929), the steady-state cosmology (Bondi \& Gold, 1948; Bondi, 1960; Hoyle, 1948), the plasma universe (Alfven, 1966; Klein, 1971; Lerner, 1991), and the theory of discordant redshift association (Field et al., 1973; Arp, 1987; Arp et al., 1990; Arp, 2003), and the Dispersive Extinction Theory (DET) (Wang, 2005, 2007, 2008, 2011).

The tired-light theory postulates that photons would get tired from the long travel and lose its energy. It would increase its wavelength and result in redshift. Such ad hoc hypothesis was not borne out by experimental evidence.

The plasma cosmology was based on the assumption that our galaxy was created from certain metagalaxy consisting of equal matter and antimatter. It was further postulated that such mixture of matter-antimatter would move through certain magnetic field, and the matter and antimatter could be separated into clouds and anti-clouds. The clouds and anti-clouds would attract and collapse under the gravitational force, leading to an explosion over a vast region hundreds of millions of light years across over a time period of hundreds of millions of years. The problem is, the mixture of matter-antimatter may not be adequately described by plasma physics since the positive and negative charges in a laboratory plasma do not annihilate. They simply neutralize each other. Plus, the existence of the original matter-antimatter and the magnetic field is highly hypothetical.

Arp's theory of discordant redshift association proclaims that the cosmic redshift does not indicate recession velocity and is not necessarily reliable distance indicator. The majority of astronomers take a skeptical view of 
Arp's interpretations of his photographs of the discordant redshift associations based on the possibility of chance alignment along the direction of sight of the objects at great distances. However, the number of Arpian objects is too large for these objects to be dismissed in entirety as chance alignments. Logically speaking, we need only a single genuine discordant association to dismiss the redshift as reliable indicator of velocity. Arp's position is supported by the abnormal redshifts of the quasars. For instance, the spectrum of the quasar $0149+335$ shows drastically different red shifts for different lines: 0.82 for $\mathrm{Mg}^{+}, 1.95$ for $\mathrm{C} 3+$; and 2.14 for $\mathrm{H}$ Lya line (Silk, 1989). The weakness with Arp's proclamation is that it does not suggest a mechanism to explain the cosmic redshift, and the abnormal redshifts of quasars.

The steady-state cosmology assumes that the mass density of universe is spatially homogeneous and isotropic on average and constant in time, but it also assumes that the universe is expanding. It further assumes constant spontaneous creation of matter to make up for the decrease of mass density due to expansion. Such theory needs nothing less than creationism. Moreover, it needs to explain why the rate of creation happens to match exactly the rate of density reduction due to expansion.

These alternatives did not gain enough support to upset the Big Bang theory. It was mainly due to the monopoly of cosmological community by the Big Bang cosmology, and partially duo to the fact that these alternatives had their own Achilles heels-the ad hoc hypotheses of some sort. It is therefore highly desired to have an alternative theory of the cosmic redshift that is free of the ad hoc hypotheses. The Dispersive Extinction Theory (DET) was proposed to answer that call (Wang, 2005, 2007, 2008, 2011).

Why a different theory of redshift would mean different cosmology? The reason is simple: The correlation between cosmic redshift and the astronomical distance is the only direct experimental evidence the Big Bang cosmologists love to quote to support a finite expanding universe if, and only if, the redshift is interpreted as due to the recession movement of the galaxies. If, however, the cosmic redshift is not caused by the recession movement of heavenly bodies, all the argument of an expanding universe is invalid, so would be the Big Bang theory. Traditionally, as a scientific theory, the Big Bang theorists should be obliged to prove that the Doppler effect is the only possible cause for cosmic redshift. But strangely enough, the burden of proof somehow shifted from the Big Bang theorists to the physics community to disprove the Doppler-shift interpretation. Unless the scientific community can come up with a good explanation of the redshift, the Big Bang theory seems to be automatically vindicated in spite of the multitude of the fundamental inconsistencies and paradoxes. It is then paramount to find the real cause for the cosmic redshift.

\section{The Dispersive Extinction Theory (DET) of Cosmic Redshift}

The Dispersive Extinction Theory, first published in Physics Essays in 2005 (Wang, 2005), attributes the cosmic redshift to the dispersive extinction (absorption plus scattering) of star light by the space medium. The extinction by interstellar medium (ISM) is generally recognized in the study of star reddening which shows that the interstellar extinction is wavelength dependent (Field, 1986; Johnson, 1968; Nathusm 1990; Ostlie \& Carroll, 1996). It was found that the blue light is absorbed and scattered more than the red component. The atmosphere behaves in the same way and causes the sky to look blue. We have good reason to believe that the intergalactic medium, or space medium, should have same dispersion characteristics as the interstellar medium. Namely, the blue component of a spectral line is extinct more than the red component.

A spectral line is not an infinitely thin line, but a Gaussian curve with certain line width. After passing through the space medium, a spectral line would suffer extinction due to absorption and scattering, and the loss is wavelength dependent, or dispersive. The blue component would be extinct more than the red component. As a result, the peak of the Gaussian curve would be shifted towards the red, causing red shift (Figure 4). The intensity will also be reduced as a result of extinction. The longer the distance of travel, the more extinction the light beam would suffer, and the greater would be the redshift. 


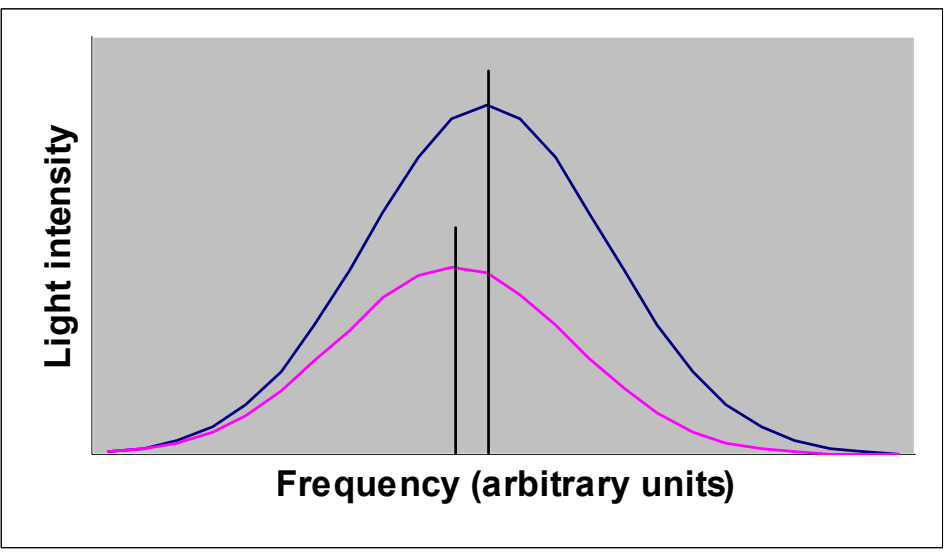

Figure 4. The redshift due to dispersive extinction by space medium. The vertical lines indicate the peaks of the original and the eventual Gaussian curves

The relationship between redshift and distance also depends on the optical density of the space medium. We must distinguish two scenarios in this regard: the unsaturated extinction in which the light intensity is weak; and the saturated extinction in which the light intensity is high enough to saturate the extinction. In the unsaturated scenario, the extinction is proportional to both the light intensity and the distance; while in the saturated scenario, the extinction depends only on the distance, not the beam intensity.

The amplitude of the original Gaussian spectral line can be described by a function:

$$
F(\omega)=F_{0} \exp \left[-\frac{\left(\omega-\omega_{0}\right)^{2}}{\gamma^{2}}\right]
$$

where $F(\omega)$ is the amplitude, $\omega_{0}$ the central frequency and $\gamma$ the standard deviation. After passing through a layer of unsaturated space medium, the above frequency distribution function is modified to become:

$$
F(\omega, r)=F_{0} \frac{r_{0}}{r} \exp \left[-\alpha r-\frac{\left(\omega-\omega_{0}\right)^{2}}{\gamma^{2}}\right]
$$

With

$$
\alpha=a+b \omega
$$

where $\alpha$ is the extinction coefficient, $a$ the non-dispersive extinction coefficient, $b$ the dispersion coefficient. $r_{0}$ is the radius of the star or galaxy an $r$ the distance from it to the earth. The factor $\left(r_{0} / r\right)$ reflects the inverse square law, while the factor $\exp [-\alpha \mathrm{r}]$ is due to the dispersive extinction by the space medium.

It can be shown (Wang, 2005) that the new spectrum Equation (9) has a new peak at the frequency:

$$
\omega_{0}^{\prime}=\omega_{0}-\frac{b \gamma^{2} r}{2}
$$

which would yield a redshift of (Wang, 2005)

$$
z=9 b c \frac{\delta \lambda^{2}}{\lambda^{3}} r
$$

where $z$ is the redshift, $c$ the speed of light, $\delta \lambda$ and $\lambda$ are the line width and wavelength.

The above equation shows that the red shift is not only linearly proportional to the distance, which agrees with Hubble law, but also proportional to the square of the line width, and inversely proportional to the cube of the wavelength. Namely, the red shifts of different spectral lines from the same heavenly body ( $r$ being the same) can be very different, depending on the line widths and the wavelength. This is the major difference between DET theory and the Hubble law (Hubble, 1926, 1929): 


$$
z=H r
$$

Comparison of Equations (12) and (13) shows that the Hubble constant $H$ has actually swallowed the line width and wavelength dependence. It explains the crudeness of Hubble law. (The data points of the plot fill almost one half of the first quadrant). If we define a new parameter Wangian:

$$
W=\frac{z \lambda_{0}^{3}}{\delta \lambda^{2}}
$$

Then

$$
W=9 c b r
$$

Equation (15) shows that it should be the $W$ function, instead of red shift $\mathrm{z}$, that is linearly proportional to the cosmic distance. If we plot the Wangian $W$ versus distance $r$, the correlation should be much better than a plot of redshift versus distance. This difference can be used as an experimental test of DET versus Big Bang.

It is quite interesting that Hubble himself did not like the Doppler-shift interpretation of the cosmic redshift because it led to a notion of expanding universe, which was quite offensive to the then well accepted scientific concept of an infinite and stable universe. Unfortunately, the Doppler effect was then the only mechanism he knew to be able to cause a change of frequency and wavelength. If DET was available then, it is not hard to imagine that Hubble might espouse himself with DET instead of the Doppler-effect interpretation. The cosmology would have followed a quite different approach. But history took its own prerogative to allow dominance of cosmology by a geocentric Big Bang theory. Guided by the Doppler-shift theory, the experimental astrophysicists did not think that the redshift would have anything to do with the line width and the wavelength. The different redshifts from the same heavenly object were simply averaged out. We tried to dig out information of the line width and wavelength dependence of redshifts from the published literature, only to be defeated everywhere. The author ventures to guess that some data containing the linewidth and wavelength dependence might be buried in the dusty archives of some lab logbooks.

\section{The Saturated Dispersive Extinction}

The scenario dealt with in the last section is a one in which the extinction by the space medium is not saturated, which is the case in the last part of travel when the star light is very weak. In the first part of the journey when the light intensity is very high, the extinction is expected to be saturated. In such scenario the frequency distribution function, Equation (8), is modified in a different way from that expressed in Equation (9):

$$
F(\omega, r)=F_{0} \frac{r_{0}}{r} \exp \left[-\frac{\left(\omega-\omega_{0}\right)^{2}}{\gamma^{2}}\right]-(p+q \omega)
$$

where $p$ and $q$ are, respectively, the saturated non dispersive and dispersive extinction constants. Straightforward calculation shows that the redshift is related to the distance as (Wang, 2008):

$$
r=\left(\frac{A_{0} r_{0}^{2} \lambda_{0}^{3}}{\pi c q \gamma_{\lambda}^{2}}\right)^{\frac{1}{3}} z^{\frac{1}{3}} \exp \left[-\frac{\lambda_{0}^{2} z^{2}}{3 \gamma_{\lambda}^{2}}\right]
$$

where $\mathrm{c}$ is the speed of light, and $\gamma_{\lambda}$ the line width in wavelength.

Equation (17) shows that saturated dispersive extinction is not linearly proportional to the distance, which differs from what indicated by the linear Hubble law. As the linearity of Hubble law is only loosely demonstrated and widely challenged by experimentalists (Segal \& Nicoll, 1992), a non linearity is quite interesting.

For small redshift, the exponential factor is nearly unity and Equation (17) becomes:

$$
z=\frac{\pi q c \sigma_{\lambda}^{2}}{A_{0} \lambda_{0}^{3}} \frac{r^{3}}{r_{0}^{2}}
$$


Equation (18) indicates that the redshift is proportional to the square of the line width and inversely proportional to the cube of the wavelength, in exactly the same way as it is in the case of unsaturated DET. It also shows that the redshift by the saturated extinction is proportional to the cube of the distance.

\section{Experimental Tests of DET versus Big Bang}

Strong Experimental evidences abound against the Big Bang theory with respect to the multitude of fundamental difficulties discussed in section 1 , such as the geocentric nature, the creation of enormous mass and energy, the horizon problem, the violation of Maxwell's speed distribution, the extreme instability of the model and the dark mass hypothesis. DET is free of any of these problems.

One of the direct evidence against the Big Bang theory is the abnormal redshifts of quasars, which is consistent with DET. The Big Bang theory predicts that the red shifts of different spectral lines from the same heavenly body must be the same (with possible small difference due to rotation). But this is in directly contradiction with the measured redshifts of quasars. For example, the quasar 0149+335 shows drastically different red shifts for different lines: 0.82 for $\mathrm{Mg}^{+}, 1.95$ for $\mathrm{C}^{3+}$; and 2.14 for H Lya line (Silk, 1989). Such huge differences could in no way be explained by the rotation of quasars.

DET offers a natural explanation to the abnormal redshifts of the quasars. Since the redshift is proportional to the square of the line width and all quasars typically have large line widths. It explains why the quasars usually have large redshifts.

\section{Detailed Test of DET}

Although DET is free of the fundamental problems and ample evidences exist in support of DET against Big Bang theory, we still look for detailed tests to further check the specific predictions of DET and prove the theory beyond reasonable doubt. Fortunately, such detailed litmus-tests do exist.

\subsection{The Correlation Test of the Wangian-Distance Plot}

The red shift-magnitude plot according to Hubble law has very poor correlation and the Hubble constant cannot be accurately determined to within a factor of two. As pointed out in Equations (14) and (15), the Wangian $W$, instead of redshift, is truly linearly dependent on the distance if the space medium is homogeneous. A $W$-r plot is expected to show better correlation than the z-r plot, and can be used to test DET versus the Doppler-effect interpretation of Hubble law.

\subsection{The Line width Dependence Test}

If we define

$$
X \equiv \frac{z \lambda^{3}}{9 c b r}
$$

Equation (15) can be written as

$$
X=\delta \lambda^{2}
$$

A plot of $X$ versus $\delta \lambda$ should show a quadratic relation.

\subsection{The Wavelength Dependence Test}

Likewise, if we define

$$
Y \equiv \frac{z}{9 c b r \delta \lambda^{2}}
$$

we have from Equation (15)

$$
Y=\frac{1}{\lambda^{3}}
$$

A plot of $Y$ versus $\lambda$ should show an inverse cubic relationship.

Equations (19) and (20) are based on the fact that the redshift is proportional to the square of the line width and inversely proportional to the cube of the wavelength, as predicted by both the saturated and unsaturated DET.

All these tests are straightforward and can be easily carried out by students. For the research groups equipped with a decent telescope and spectrometer, it is merely a repetition of the past red-shift measurements. The only extra work is to record the wavelength and line width of each spectral line. The meager cost will prove worth spending considering the significance of having a correct theory of cosmology. 


\section{A Possible Explanation of the Abnormal Redshifts of Quasars}

DET offers a possible explanation for the abnormal redshifts of quasars: It is probably due, at least partially, to the large line width typical of the quasars. But the story does not end there. DET even suggests that the reported large redshifts of quasars might be due to erroneous identification! To see this, let us consider two spectral lines of certain quasar having wavelengths $\lambda_{1}$ and $\lambda_{2}$. After passing through the space medium, these wavelengths have shifted to $\lambda_{1}$ ' and $\lambda_{2}$ '. According to Equation (12), the redshifts of these two lines are, respectively,

$$
\left\{\begin{array}{l}
z_{1}=\frac{\lambda_{1}{ }^{\prime}-\lambda_{1}}{\lambda_{1}}=9 b c \frac{\delta \lambda_{1}^{2}}{\lambda_{1}^{3}} r \\
z_{2}=\frac{\lambda_{2}{ }^{\prime}-\lambda_{2}}{\lambda_{2}}=9 b c \frac{\delta \lambda_{2}{ }^{2}}{\lambda_{2}{ }^{3}} r
\end{array}\right.
$$

Due to the fact that the wavelengths and the line widths are different for the two spectral lines, their redshifts are different. Namely, $z_{1}$ is generally not equal to $z_{2}$. Since

$$
\left\{\begin{array}{l}
\lambda_{1}{ }^{\prime}=\left(z_{1}+1\right) \lambda_{1} \\
\lambda_{2}{ }^{\prime}=\left(z_{2}+1\right) \lambda_{2}
\end{array}\right.
$$

Which means,

$$
\frac{\lambda_{1}^{\prime}}{\lambda_{2}^{\prime}} \neq \frac{\lambda_{1}}{\lambda_{2}}
$$

Equation (23) shows that the ratio between the red-shifted wavelengths, $\left(\lambda_{1}{ }^{\prime} / \lambda_{2}{ }^{\prime}\right)$, is in general not equal to the ratio between the original wavelengths, $\left(\lambda_{1} / \lambda_{2}\right)$. However, the basic assumption for identification of the spectral lines is that the ratio between the wavelengths does not change. The experimentalists then would look for the elements with spectral lines in the visible range having the ratio of wavelengths given by $\left(\lambda_{1}{ }^{\prime} / \lambda_{2}{ }^{\prime}\right)$. Suppose thus identified wavelengths are $\lambda_{2}$ " and $\lambda_{1}$ ", which are quite different from the truly original wavelengths, the redshift would be reported as

$$
z^{*}=\frac{\lambda_{1}^{\prime}-\lambda_{1}^{\prime \prime}}{\lambda_{1}^{\prime \prime}}
$$

The identified redshift $\mathrm{z}^{*}$ of Equation (24) could be much greater than the real redshift of Equation (22). It would yield a much greater redshift $z^{*}$.

\section{Acknowledgements}

The author wishes to thank Ms. Heather DeLancett for her technical editing assistance in preparing the manuscript.

\section{References}

Abell, G. O. (1958). The Distribution of Rich Clusters of Galaxies. Astrophys. J. Suppl., 3, 211-228. http://dx.doi.org/10.1086/190036

Ahmed, Z., Akerib, D. S., Arrenberg, S., Bailey, C. N., Balakishiyeva, D., Baudis, L., ... Zhan, J. (2009). Results from the Final Exposure of the CDMS II Experiment. Science, 327, 1619-1621. http://dx.doi.org/10.1126/science.1186112

Alfven, H. (1966). Worlds-Antiworlds. San Francisco: Freeman. Retrieved from http://www.archive.org/details/WorldsAntiworlds

Arp, H. C. (1987). Quasars, Redshifts, and Controversies, Berkeley, CA: Interstellar Media.

Arp, H. C. (2003). Catalogue of discordant redshift associations. Montreal, Quebec, Canada: Apeiron. 
Arp, H. C., Burbidge, G., Hoyle, F., Narlikar, J. V., \& Wishramasinghe, N. C. (1990). The extragalactic Universe: an alternative view. Nature, 346, 807-812. Retrieved from http://www.nature.com/nature/journal/v346/n6287/abs/346807a0.html

Ashtekar, A, Baez, J., Corichi, A., \& Krasnov, K. (1998). Quantum Geometry and Black Hole Entropy. Physical Review Letters, 80, 904-907. http://dx.doi.org/10.1103/PhysRevLett.80.904

Ashtekar, A. (1986). New variables for classical and quantum gravity. Physical Review Letters, 57, 2244-2247. http://dx.doi.org/10.1103/PhysRevLett.57.2244

Ashtekar, A. (1987). New Hamiltonian formulation of general relativity. Physical Review D, 36, 1587-1602. http://dx.doi.org/10.1103/PhysRevD.36.1587

Ashtekar, A., \& Lewandowski, J. (2004). Background Independent Quantum Gravity: A Status Report. Classical and Quantum Gravity, 21, R53-R152. http://dx.doi.org/10.1088/0264-9381/21/15/R01

Bertone, G., \& Merritt, D. (2005). Dark Matter Dynamics and Indirect Detection. Modern Physics Letters A, 20(14), 1021-1036. http://dx.doi.org/10.1142/S0217732305017391

Bertone, G., Hooper, D., \& Silk, J. (2005). Particle dark matter: Evidence, candidates and constraints. Physics Reports, 405(5-6), 279-390. http://dx.doi.org/10.1016/j.physrep.2004.08.031

Bondi, H. (1960). Cosmology (2nd ed., 166). Cambridge, U.K.: Cambridge University Press.

Bondi, H., \& Gold, T. (1948). The Steady-State Theory of the Expanding Universe, M.N.R.A.S., 108, 252. Retrieved from http://adsabs.harvard.edu/cgi-bin/bib_query?1948MNRAS.108..252B

Davis, M., Efstathiou, G., Frenk, C. S., \& White, S. D. M. (1985). The evolution of large-scale structure in a universe dominated by cold dark matter. Astrophysical Journal, 292(1985), 371-394. http://dx.doi.org/10.1086/163168

De Vaucouleurs, G. (1993). The extragalactic distance scale. VIII. A comparison of distance scales. Astrophys. J., 415, 10-32. http://adsabs.harvard.edu/doi/10.1086/173138

Dicke, R. H., Peebles, P. J. E., Roll, P. G., \& Wilkinson, D. T. (1965). Cosmic Black-Body Radiation. Astrophys. J., 142, 414-419. http://adsabs.harvard.edu/doi/10.1086/148306

Field, G. B. (1986). Theory of the Interstellar Medium in Highlights of Modern Astrophysics, Concepts and Controversies (pp235-265). In S. L. Shapiro and S.A. Teukolsky (Ed.). New York, NY: Wiley

Field, G. B., Arp, H. C., \& Bahcall, J. N. (1973). The Redshift Controversy. Reading, MA: W.A. Benjamin.

Green, M. B., \& Schwarz, J. H. (1984). Anomaly Cancellations in Supersymmetric D=10 Gauge Theory and Superstring Theory. Physics Letters B, 149, 117-122. http://dx.doi.org/10.1016/0370-2693(84)91565-X

Hawking, S. (1993). Black Holes and Baby Universes and other Essays (p97). New York, NY: Bantam Books.

Hoessel, J. G., Gunn, J. E., \& Thuan, T. X. (1980). The photometry properties of brightest cluster galaxies. I. Absolute magnitudes in 116 nearby Abell clusters. Astrophys. J., 241, 486-492. http://dx.doi.org/10.1086/158363

Hoyle, F. (1948). A New Model For The Expanding Universe. M.N.R.A.S., 108, 372. Retrieved from http://articles.adsabs.harvard.edu/full/1948MNRAS.108..372H

Hubble, E. (1926). Extra-galactic Nebulae. Astrophys. J., 64, 321. http://dx.doi.org/10.1086/143018

Hubble, E. (1929). A Relation between Distance and Radial Velocity among Extra-Galactic Nebulae. Proceedings of the National Academy of Sciences of the United States of America, 15(3), 168-173. http://dx.doi.org/10.1073/pnas.15.3.168

Hubble, E. (1936). The Realm of the Nebulae. New Haven, CT: Yale University Press.

Johnson, H. L. (1968). Interstellar Extinction in Nebulae and Interstellar Matter. In B. M. Middlehurst, \& L. H. Aller, (Eds.). Chicago, IL: The University of Chicago Press.

Kane, G., \& Watson, S. (2008). Dark Matter and LHC: what is the Connection? Modern Physics Letters A, 23, 2103-2123. http://dx.doi.org/10.1142/S0217732308028314

Klein, O. (1971). Arguments Concerning Relativity and Cosmology. Science, 171, 339. http://dx.doi.org/10.1126/science.171.3969.339 
Lerner, E. J. (1991). The Big Bang Never Happened. New York, NY: Vintage Books.

Mathis, J. S. (1990) Interstellar Dust and Extinction. Annual Review of Astronomy and Astrophysics, $28,37$. http://dx.doi.org/10.1146/annurev.aa.28.090190.000345

Ohanian, H. C. (1976). Gravitation and Spacetime. New York, NY: W.W. Norton \& Co., Inc.

Ostlie, D. A., \& Carroll, B. W. (1996). An Introduction to Modern Stellar Astrophysics (pp. 437-44). Reading, MA: Addison-Wesley.

Rovelli, C. (1996). Black Hole Entropy from Loop Quantum Gravity. Physical Review Letters, 77, 3288-3291. http://dx.doi.org/10.1103/PhysRevLett.77.3288

Rovelli, C., \& Smolin, L. (1988). Knot theory and quantum gravity. Physical Review Letters, 61, 1155. http://dx.doi.org/10.1103/PhysRevLett.61.1155

Rovelli, C., \& Smolin, L. (1995). Discreteness of area and volume in quantum gravity. Nucl. Phys. B, 442, 593-622. http://dx.doi.org/10.1016/0550-3213(95)00150-Q

Rubin, V. C., \& Ford, W. K. Jr. (1970). Rotation of the Andromeda Nebula from a Spectroscopic Survey of Emission Regions. The Astrophysical Journal, 159, 379. http://dx.doi.org/10.1086/150317

Sandage, A., \& Tammann, G. A. (1990). Steps toward the Hubble constant. IX - The cosmic value of H(0) freed from all local velocity anomalies. Astrophys. J., 365, 1-12. http://dx.doi.org/10.1086/169453

Segal, I. E., \& Nicoll, J. F. (1992). Apparent nonlinearity of the redshift distance relation in infrared astronomical satellite galaxy samples. Proc. Natl. Acad. Sci. Astronomy, USA, 89(24), 11669-11672. http://dx.doi.org/10.1073/pnas.89.24.11669

Silk, Joseph. (1989). The Big Bang (pp. 259-263). New York, NY: W.H. Freeman and Company.

Smolin, L. (1990). Loop space representation of quantum general relativity. Nuclear Physics B, 331, 80-152. http://dx.doi.org/10.1016/0550-3213(90)90019-A

Smolin, L. (2006). The Case for Background Independence. In Dean Rickles, et al. (eds.), The Structural Foundations of Quantum Gravity (p196ff). http://dx.doi.org/10.1093/acprof:oso/9780199269693.001.0001

Smolin, L. (2010). Newtonian gravity in loop quantum gravity. Perimeter Institute for Theoretical Physics. Retrieved from arXiv:1001.3668v2 [gr-qc]

Tammann, G. A., \& Sandage, A. (1985). The infall velocity toward Virgo, the Hubble constant, and a search for motion toward the microwave background. Astrophys. J., 294, 81. http://dx.doi.org/10.1086/163277

Thiemann, T. (2003). Lectures on Loop Quantum Gravity. Lectures Notes in Physics, 631, 41-135. http://dx.doi.org/10.1007/978-3-540-45230-0_3

Thiemann, T. (2006). Loop Quantum Gravity: An Inside View. In Ion-Olimpiu Stamatescu \& Erhard Seiler (Eds). Approaches to Fundamental Physics. Lecture Notes in Physics, 721, Part V, 185-263. http://dx.doi.org/10.1007/978-3-540-71117-9_10

Wang, L. J. (2005). The Dispersive Extinction Theory of Red Shifts. Physics Essays, 18(2), 177-181. http://dx.doi.org/10.4006/1.3025736

Wang, L. J. (2007). On the Geocentric Nature of Hubble Law. Physics Essays, 20(2), 329. http://dx.doi.org/10.4006/1.3119435

Wang, L. J. (2008). An Experimental Method to Test DET. Physics Essays, 21(3), 233-237. http://dx.doi.org/10.4006/1.3028141

Wang, L. J. (2011). Saturated Dispersive Extinction Theory of Redshift. Physics Essays, 24(4), 2011. http://dx.doi.org/10.4006/1.3651528

Witten, E. (1995). Some problems of strong and weak coupling. Future Perspectives in String Theory. Los Angeles, CA, University of Southern California: March 13-18, 1995. http://physics.usc.edu/Strings95/Proceedings/index.html\#witten arxiv.org/abs/hep-th/9507121

Yi, L., \& Lee, S. (1999). Supersymmetric Spin Networks and Quantum Supergravity. Physical Review D, 61, 044008. http://dx.doi.org/10.1103/PhysRevD.61.044008

Zwicky, F. (1929). On the Red Shift of Spectral Lines through Interstellar Space. PNAS, 15, 773. http://dx.doi.org/10.1073/pnas.15.10.773 
Zwicky, F. (1933). Die Rotverschiebung von extragalaktischen Nebeln. Helvetica Physica Acta, 6, $110-127$. Retrieved from: http://articles.adsabs.harvard.edu/full/1933AcHPh...6..110Z

Zwicky, F. (1937). On the Masses of Nebulae and of Clusters of Nebulae. The Astrophysical Journal, 86, 217. http://dx.doi.org/10.1086/143864 